\title{
DESENVOLVIMENTO DE UMA METODOLOGIA RÁPIDA E DE BAIXO CUSTO PARA QUANTIFICAÇÃO DE ACETONA- BUTANOL-ETANOL EM PROCESSOS FERMENTATIVOS INDUSTRIAIS
}

\author{
L. J. VISIOLI ${ }^{1}$, E. A. ALVES ${ }^{2}$, A. C. M. TRINDADE ${ }^{2}$, R. C. $\mathrm{KHUN}^{2}$, M. SCHWAAB ${ }^{2}$, M. A. \\ MAZUTTI $^{2}$ \\ ${ }^{1}$ Universidade Estadual de Maringá, Departamento de Engenharia Química \\ ${ }^{2}$ Universidade Federal de Santa Maria, Departamento de Engenharia Química \\ E-mail para contato: luizjvisioli@gmail.com
}

\begin{abstract}
RESUMO - Biobutanol obtido por fermentação clostridial representa um importante biocombustível. Neste cenário, desenvolveu-se um método baseado na densidade da mistura para determinação da concentração dos solventes acetona, butanol e etanol (ABE) como alternativa ao uso de métodos baseados em cromatografia. Para esta proposta, diversos pontos de calibração foram preparados usando diferentes meios de cultura para representar condições reais para a fermentação clostridial. As densidades das misturas foram determinadas para concentrações de $A B E$ variando entre 0 e $25 \mathrm{~g} / \mathrm{L}$, em três diferentes níveis de concentração de açúcar para representar a evolução do meio durante a fermentação. Os resultados obtidos demonstram uma relação linear da variação da densidade com as concentrações de $\mathrm{ABE}$ e açúcares. A principal contribuição deste trabalho foi a determinação da concentração dos solventes $\mathrm{ABE}$ com uma metodologia simples, rápida e de baixo custo.
\end{abstract}

\section{INTRODUÇÃO}

A produção de biocombustíveis é uma das chaves para o desenvolvimento de uma economia sustentável (Oudshoorn et al., 2009). Etanol é o biocombustível mais usado no mundo, no entanto, o butanol apresenta melhores propriedades, principalmente a energia específica, que é significativamente maior que a do etanol (Al-Shorgani et al., 2012). O biobutanol pode ser atrativo, ainda, para substituição do uso de gasolina, já que, apresenta grande similaridade com este combustível fóssil (Mayank et al., 2013). Além disso, biobutanol pode ser obtido a partir de diferentes matérias primas incluindo diversos resíduos de indústria de alimentos e agricultura (Qureshi et al., 2007).

A produção do biobutanol tem estado em evidência nos últimos anos. De acordo com Qureshi et al. (2007) há uma tendência de crescimento rápido na produção de biobutanol, principalmente em países em acelerado desenvolvimento como, por exemplo, China e Brasil. Por outro lado, as pesquisas tem se direcionado para a utilização da biomassa lignocelósica para a produção industrial de butanol. Esta matéria prima tem potencial para reduzir o custo do biocombustível (Jurgens et al., 2012). A importância do biobutanol como combustível 


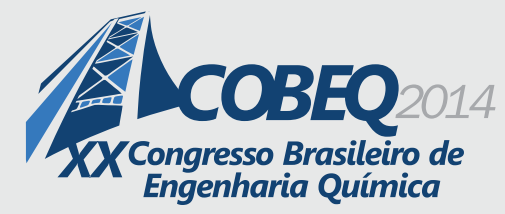

alternativo pode ser observado pelo grande número de patentes registradas nos últimos anos em todo o mundo (Visioli et al., 2013).

A perspectiva de expansão na produção de biobutanol gera a necessidade de um método eficiente e barato para quantificação dos produtos no mosto de fermentação. Estes produtos são butanol (principal), etanol e acetona (fermentação ABE) presente em diferentes títulos dependendo da cepa utilizada (Napoli et al., 2010). Atualmente esta determinação é feita utilizando a cromatografia gasosa (Liu et al., 2010; Lépiz-Aguilar et al., 2011) na maior parte dos estudos. No entanto, a determinação de butanol usando cromatografia é limitada para aplicações industriais devido ao custo associado com os equipamentos, acessórios e produtos químicos.

Uma alternativa, de menor custo efetivo, é o uso da variação da densidade do meio para determinação dos produtos. A densidade é uma propriedade física, fácil de mensurar, e que apresenta valores muito diferentes entre produtos e caldo de fermentação inicial. Os solventes ABE tem densidade de $0,8 \mathrm{~g} / \mathrm{cm}^{3}$ aproximadamente, já o mosto tem densidade, antes do início da fermentação, maior do que $1,0 \mathrm{~g} / \mathrm{cm}^{3}$ (densidade da água + sólidos dissolvidos). Isso mostra uma diferença maior do que $20 \%$ entre a densidade dos solventes e do meio de fermentação, sendo então, possível associar o decréscimo na densidade do meio com a produção de solventes em um método simples e rápido.

Tendo em vista estes aspectos, o objetivo principal deste trabalho é desenvolver e validar uma metodologia para determinar a concentração dos solventes $\mathrm{ABE}$ baseada na variação da densidade do meio de fermentação. Na primeira etapa do trabalho, soluções com diferentes concentrações de $\mathrm{ABE}$ são preparadas, utilizando água como solvente, para os testes iniciais do comportamento da densidade. Na segunda etapa, diferentes concentrações de ABE são diluídas em meio RCM e sorgo sacaríneo, com diferentes concentrações de açúcar, para simular o processo fermentativo real.

\section{MATERIAIS E MÉTODOS}

\subsection{Produtos químicos}

Os solventes (acetona, etanol e butanol) e demais produtos químicos usados eram da Sigma-Aldrich ${ }^{\circledR}$ e todos com pureza PA. O sorgo sacaríneo foi gentilmente fornecido pela microdestilaria da Universidade Federal de Santa Maria e o mesmo foi cultivado sob as coordenadas geográficas $29^{\circ} 41^{\prime} 29^{\prime \prime}$ sul, $53^{\circ} 48^{\prime} 3^{\prime \prime}$ oeste.

\subsection{Determinação de ABE em água}

Pontos de calibração foram preparados em concentrações variando de 0 a $25 \mathrm{~g} / \mathrm{L}$ para cada um dos solventes individualmente. Depois todos os solventes foram misturados de acordo com a razão molar 6:3:1 para butanol, acetona e etanol, respectivamente. Mais tarde, amostras com concentrações variando de 0 a $25 \mathrm{~g} / \mathrm{L}$ da solução de solventes $\mathrm{ABE}$ foram preparadas. Para cada 


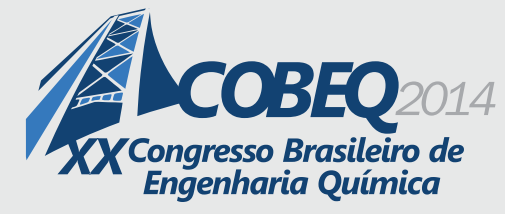

ponto de calibração, foi determinada a densidade da solução de água e solventes, assim como de uma amostra de água pura. A diferença entre os valores medidos para a densidade da mistura e a da água pura foi usada na calibração conforme Equação 1.

$$
A B E=a+b \cdot \Delta \rho
$$

Onde $A B E$ é a concentração de solventes em $(\mathrm{g} / \mathrm{g}), \triangle \rho$ é a diferença de densidades entre água e a solução contendo os solventes $\left(\mathrm{g} / \mathrm{cm}^{3}\right)$ e $a, b$ são os parâmetros do modelo linear a ser estimados.

\subsection{Determinação de ABE em meio RCM}

Para representar uma condição real de fermentação, solventes $A B E$ foram misturados, respeitando a mesma proporção molar 6:3:1 para butanol, acetona e etanol, respectivamente, em Reinforced Clostridium Medium (RCM) (Khamaiseh et al., 2012), o qual contém (g/L): extrato de levedura $(3,0)$, extrato de carne $(10,0)$, peptona $(10,0)$, amido solúvel $(1,0)$, , hidrocloreto de L-cisteina $(0,5)$, acetato de sódio $(3,0)$, agar $(0,5), \mathrm{NaCl}(5,0)$. Depois, amostras com concentrações variando entre 0 a $25 \mathrm{~g} / \mathrm{L}$ de solventes foram preparadas e a densidade foi determinada. Nesta etapa do trabalho, foi verificado o efeito da concentração de açúcar no processo e observado se ainda era possível determinar a concentração de $A B E$, já que, a influência da concentração de açúcar é grande na variação da densidade da solução e esta varia durante o processo fermentativo. Desta forma, se torna necessário o conhecimento da concentração de açúcar para ser possível predizer o valor da concentração de ABE. Com esta finalidade, as amostras foram preparadas usando três diferentes concentrações de açúcar; 0,30 e $60 \mathrm{~g} / \mathrm{L}$, usando glicose anidra. Da mesma maneira descrita anteriormente a densidade foi determinada e a diferença entre a medida sem adição de solventes e as com diferentes concentrações foram usadas para desenvolver o modelo, conforme Equação 2. Onde é acrescentado apenas um parâmetro, $b$, que multiplica a concentração de açúcar $S$.

$$
A B E=a+b \cdot S+c \cdot \Delta \rho
$$

\subsection{Determinação de ABE em sorgo sacaríneo como meio de fermentação}

Para representar uma condição real de fermentação, solventes ABE foram misturados, na mesma razão molar de 6:3:1 já descrita, usando sorgo sacaríneo como meio de fermentação com três diferentes concentrações de açúcar $(10,35$ e $60 \mathrm{~g} / \mathrm{L})$, contendo além disso em $(\mathrm{g} / \mathrm{L})$ : extrato de levedura $(2,0), \mathrm{KH}_{2} \mathrm{PO}_{4}(0,5), \mathrm{K}_{2} \mathrm{HPO}_{4} .3 \mathrm{H}_{2} \mathrm{O}(0,65), \mathrm{MgSO}_{4} .7 \mathrm{H}_{2} \mathrm{O}(0,2), \mathrm{FeSO}_{4} .7 \mathrm{H}_{2} \mathrm{O}(0,01)$, $\mathrm{MnSO}_{4} \cdot \mathrm{H}_{2} \mathrm{O}(0,01), \mathrm{NaCl}(0,01)$, tioglicolato de sódio $(1,0)$ e triptona $(1,0)$ (Liu et al., 2010). Posteriormente, as amostras com concentração de solventes variando de 0 a $25 \mathrm{~g} / \mathrm{L}$ foram preparadas e as densidades determinadas. A diferença entre os valores de densidade do sorgo sem adição de solventes e cada um dos valores com adição foi usado para ajuste dos parâmetros do modelo descrito na Equação 2. 


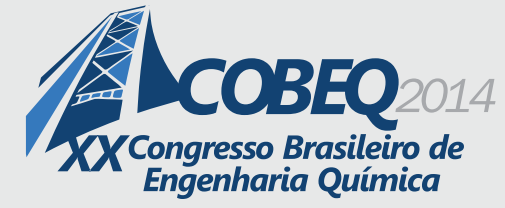

\subsection{Determinação da densidade da mistura}

As densidades das soluções eram medidas em um densímetro Anton Paar DMA 4500 equipado com correção automática da temperatura e operando em modo estático. As amostras eram injetadas em triplicata para assegurar que a alça de amostragem estivesse completamente livre de contaminação. Então as amostras eram injetadas e a seringa mantida acoplada no injetor do densímetro, para evitar a entrada de bolhas de ar. A densidade era determinada na temperatura de $20 \pm 0,01{ }^{\circ} \mathrm{C}$.

\subsection{Estimação dos parâmetros}

Todos os parâmetros dos modelos propostos foram estimados usando o algoritmo de Levenberg-Marquardt do software Statistica 7.0 (Statsoft Inc., Tulsa, OK, USA). A estimação consiste na minimização da função objetivo soma dos mínimos quadrados entre os valores preditos e experimentais.

\section{RESULTADOS E DISCUSSÃO}

\subsection{Estimação para $A B E$ em água}

Os resultados apresentados nesta seção dão suporte para as próximas etapas do trabalho, já que, demonstram a relação entre concentração de solventes e densidade. A Figura 1a mostra a influência da concentração, de cada um dos solventes separadamente, na densidade da mistura. É possível observar que um modelo linear pode descrever esta relação. O coeficiente de determinação dos três ajustes é superior a 0,999 e a probabilidade dos parâmetros serem significativos está muito próxima de $100 \%$. Além disso, uma analise visual demonstra uma boa concordância entre predição e dados experimentais.

No entanto, em situações reais de uma fermentação clostridial, onde todos os solventes (ABE) estarão dissolvidos simultaneamente no meio líquido, torna-se necessário verificar se a relação de linearidade se mantém inalterada. Por esta razão, foi testado o método para quantificar os solventes $\mathrm{ABE}$ em água considerando a proporção molar 6:3:1 e os resultados podem ser verificados na Figura 1b. A relação linear entre concentração de $A B E$ e densidade da mistura é mantida. Da mesma maneira como foi observado para o uso dos solventes de forma individual, o coeficiente de determinação é superior a 0,999 e a probabilidade dos parâmetros serem significativos é muito próxima de $100 \%$. Estes resultados aumentam a probabilidade de um modelo representar bem a produção de butanol através da variação da densidade usando um meio real durante o processo de fermentação. No entanto, este método não será capaz de predizer a concentração dos solventes separadamente, uma vez que todos eles apresentam variação similar na densidade em diferentes concentrações. Por outro lado, é possível obter uma estimativa aproximada para as concentrações de cada composto, já que, um mesmo micro-organismo produz uma razão fixa dos solventes. 

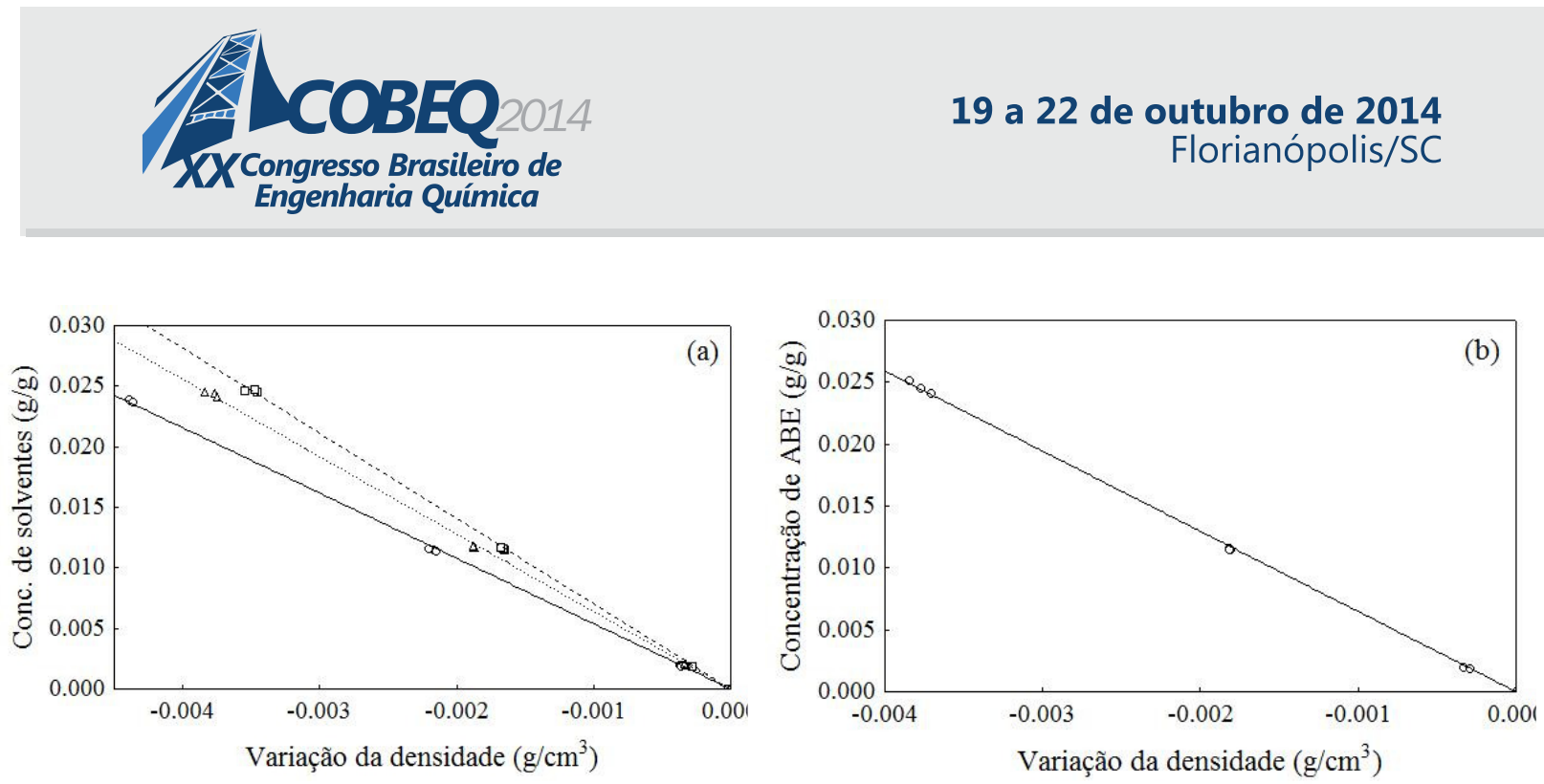

Figura 1 - Relação dos dados experimentais de densidade $\left(\mathrm{g} / \mathrm{cm}^{3}\right)$ e concentração de solventes $(\mathrm{g} / \mathrm{g})$ para (a) compostos puros (butanol $(\Delta)$, acetona $(\square)$ e etanol $(\circ)$ ) e (b) para mistura ABE em água destilada.

\subsection{Estimação de ABE em meio RCM}

Os resultados apresentados na Figura 1 são para mistura de água destilada e solventes ABE em diferentes concentrações. No entanto, para uma fermentação real é necessária a presença de outros compostos no meio. Por exemplo, se compostos sintéticos forem usados para formular um meio de cultura haverá presença de sais, e principalmente, açúcar que altera fortemente a densidade da mistura. Por esta razão, o meio utilizado passou a ser RCM, o qual já é largamente aplicado para o crescimento celular de bactérias do gênero clostridium (Knoshaug e Zhang, 2009).

Tabela 1 - Intervalo de confiança, desvio padrão, p-level, função objetivo final do ajuste dos dados experimentais usando RCM como meio e parâmetros estimados para a Equação 2

\begin{tabular}{c|c|c|c|c|c}
\hline Parâmetro & $\begin{array}{c}\text { Valor } \\
\text { estimado }\end{array}$ & $\begin{array}{c}\text { Desvio } \\
\text { padrão }\end{array}$ & p-level & $\begin{array}{c}\text { Lim. infer. } \\
\text { conf. }\end{array}$ & $\begin{array}{c}\text { Lim. super. } \\
\text { conf. }\end{array}$ \\
\hline A & 0,00124 & 0,000290 & 0,0002 & 0,00065 & 0,00184 \\
\hline B & 0,00195 & 0,000033 & $<0,0001$ & 0,00188 & 0,00202 \\
\hline C & $-5,36712$ & 0,089532 & $<0,0001$ & $-5,55082$ & $-5,18341$ \\
\hline \multicolumn{7}{c}{ Função objetivo } & \multicolumn{3}{c}{0,0000186} \\
\hline
\end{tabular}

Na Figura 2a são apresentados os valores para a densidade obtidos para diferentes concentrações de açúcar e solventes. Um ajuste linear perfeito é verificado para todos os pontos experimentais, indicando que é possível predizer a concentração de $\mathrm{ABE}$ em função da variação da densidade e da concentração de açúcar presente no meio. O coeficiente de determinação é maior que 0,99 e a probabilidade dos parâmetros serem significativos é muito próxima a $100 \%$, da mesma forma que foi observado para soluções em água. É evidente que a inserção da concentração de açúcar como uma variável a ser medida durante a análise está adequada para tornar a técnica aplicável. Os valores dos parâmetros, desvio padrão, nível de significância e intervalo de confiança são apresentados na Tabela 1. O p-level (probabilidade de o parâmetro não 


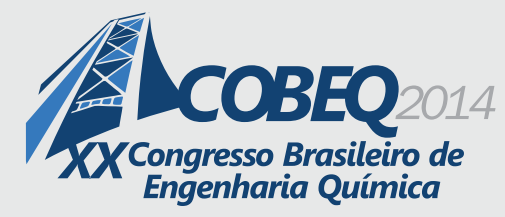

ser significativo) de cada um dos parâmetros está muito próximo de zero, indicando que os mesmos são, estatisticamente, significativos. Isso demonstra que o modelo proposto pode ser usado como uma rota analítica menos onerosa para a estimativa da produção de butanol em fermentações por clostridium.

\subsection{Estimação de ABE em sorgo sacaríneo como meio de fermentação}

Fermentações industriais são realizadas usando, preferencialmente, matérias primas de baixo custo e, por esta razão, o método proposto foi aplicado para determinação da concentração de $\mathrm{ABE}$ em meio de fermentação baseado no uso de caldo de sorgo sacaríneo (como fonte de açúcares), já que, este meio pode ser usado para fermentação butílica (Yu et al., 2012). Os pontos de calibração para diferentes concentrações de açúcares e solventes ABE eram preparados de forma similar aquela para a determinação de $\mathrm{ABE}$ em meio RCM e a variação da densidade era determinada em cada caso.

A Figura $2 \mathrm{~b}$ mostra os resultados experimentais e também o ajuste linear proposto. Como pode ser visto, há concordância entre os pontos experimentais e o modelo ajustado, mostrando que a variação da densidade e a concentração de açúcar podem ser usadas para predizer os valores da concentração de solventes $\mathrm{ABE}$ em um simples, rápido e menos oneroso método. $\mathrm{O}$ coeficiente de determinação é, novamente, maior que 0,99 .
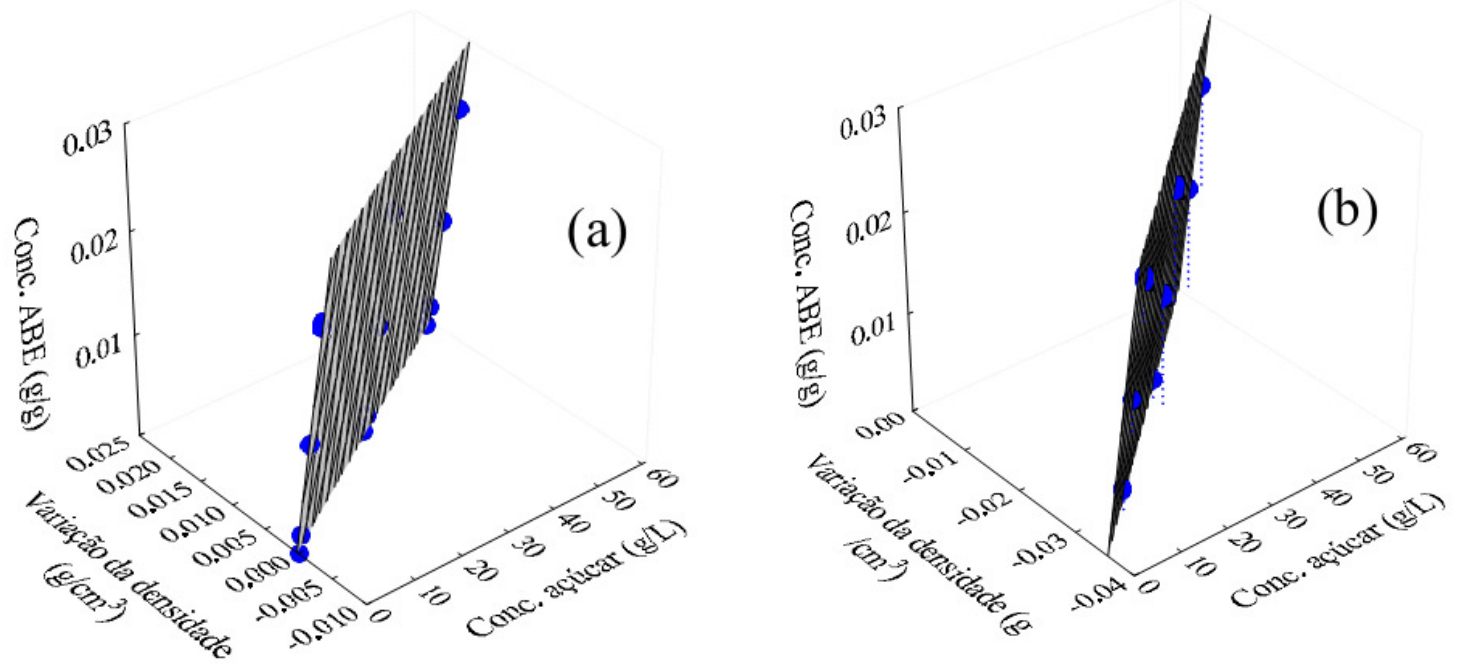

Figura 2 - Relação dos dados experimentais de variação da densidade $\left(\mathrm{g} / \mathrm{cm}^{3}\right)$, concentração de solventes ABE na proporção molar 6:3:1 (g/g), concentração de açúcar (g/L) e ajuste do modelo linear para o uso de (a) meio RCM e (b) meio sorgo sacaríneo.

Os valores dos parâmetros, desvio padrão, nível de significância e intervalo de confiança são apresentados na Tabela 2, onde fica confirmado que o modelo linear representa satisfatoriamente o comportamento dos dados experimentais. Outra importante característica demonstrada é que o parâmetro "c" para as calibrações em meio RCM e sorgo sacaríneo são 


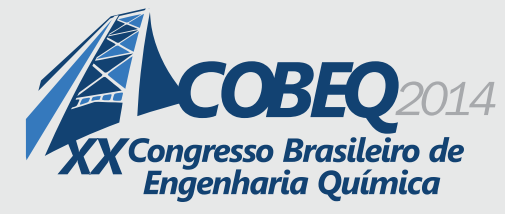

estatisticamente iguais, indicando que o decréscimo da densidade nos dois casos é bastante similar. Com isso, pode-se dizer que este parâmetro não é dependente das propriedades do meio. Assim, é esperado, que para meios similares, seja possível ter uma estimativa preliminar da produção de butanol, em uma concentração de açúcar fixa, sem necessidade de calibração do método.

Tabela 2 - Intervalo de confiança, desvio padrão, p-level, função objetivo final do ajuste dos dados experimentais usando sorgo sacaríneo como meio e parâmetros estimados para a

\section{Equação 2}

\begin{tabular}{c|c|c|c|c|c}
\hline Parâmetro & $\begin{array}{c}\text { Valor } \\
\text { estimado }\end{array}$ & $\begin{array}{c}\text { Desvio } \\
\text { padrão }\end{array}$ & p-level & $\begin{array}{c}\text { Lim. infer. } \\
\text { conf. }\end{array}$ & $\begin{array}{c}\text { Lim. super. } \\
\text { conf. }\end{array}$ \\
\hline A & $-0,19421$ & 0,005367 & $<0,0001$ & $-0,20529$ & $-0,18314$ \\
\hline B & 0,00320 & 0,000084 & $<0,0001$ & 0,00303 & 0,00337 \\
\hline C & $-5,46423$ & 0,141423 & $<0,0001$ & $-5,75611$ & $-5,17235$ \\
\hline
\end{tabular}

\section{CONCLUSÕES}

Neste trabalho foi desenvolvido um método para estimação da concentração de solventes $\mathrm{ABE}$ em meios de fermentação baseado na variação da densidade da mistura. Os resultados obtidos demonstram uma relação linear entre variação da densidade e concentração de solventes $\mathrm{ABE}$ em água. Para meios de fermentação reais, foi necessário levar em consideração a concentração de açúcares, dessa forma o método apresentou resultados satisfatórios para um meio de fermentação sintético e um industrial. A principal contribuição deste trabalho foi determinar a concentração de $\mathrm{ABE}$ por um procedimento simples, rápido e de baixo custo.

\section{REFERÊNCIAS}

AL-SHORGANI, N.K.N.; KALIL, M.S.; YUSOFF, W.M.W. Biobutanol production from rice bran and de-oiled rice bran by Clostridium saccharoperbutylacetonicum N1-4. Bioproc Biosyst Eng, v. 35, p. 817-826, 2012.

JURGENS, G.; SURVASE, S.; BEREZINA, O.; SKLAVOUNOS, E.; LINNEKOSKI, J.; KURKIJÄRVI, A.; VÄKEVÄ, M.; VAN HEININGEN, A.; GRANSTRÖM, T. Butanol production from lignocellulosics. Biotechnol Lett, v. 34, p. 1415-1434, 2012.

KHAMAISEH, E.I.; KALIL, M.S.; DADA, O.; EL-SHAWABKEH, I.; YUSOFF, W.M.W. Date fruit as carbon source in RCM-modified medium to produce biobutanol by clostridium acetobutylicum NCIMB 13357. Journal of Applied Sciences, v. 12, p. 1160-1165, 2012.

KNOSHAUG, E.P.; ZHANG, M. Butanol tolerance in a selection of microorganisms. Appl Biochem Biotech, v. 153, p. 13- 20, 2009. 
LÉPIZ-AGUILAR, L.; RODRÍGUEZ-RODRÍGUEZ, C.E.; ARIAS, M.L.; LUTZ, G.; ULATE, W. Butanol production by Clostridium beijerinckii BA101 using cassava flour as fermentation substrate: enzymatic versus chemical pretreatments. World J Microb Biot, v. 27, p. 1933-1939, 2011.

LIU, Z.; YING, Y.; LI, F.; MA, C.; XU, P. Butanol production by Clostridium beijerinckii ATCC 55025 from wheat bran. J Ind Microbiol Biotechnol, v. 37, p. 495-501, 2010.

MAYANK, R.; RANJAN, A.; MOHOLKAR, V.S. Mathematical models of ABE fermentation: review and analysis. Crit Rev Biotechnol, v. 33, p. 419-447, 2013.

NAPOLI, F.; OLIVIERI, G.; RUSSO, M.E.; MARZOCCHELLA, A.; SALATINO, P. Butanol production by Clostridium acetobutylicum in a continuous packed bed reactor. $J$ Ind Microbiol Biot, v. 37, p. 603-608, 2010.

OUDSHOORN, A.; VAN DER WIELEN, L.A.M.; STRAATHOF, A.J.J. Adsorption equilibria of bio-based butanol solutions using zeolite. Biochem Eng J, v. 48, p. 99-103, 2009.

QURESHI, N.; SAHA, B.C.; COTTA, M.A. Butanol production from wheat straw hydrolysate using Clostridium beijerincki. Bioproc Biosyst Eng, v. 30, p. 419-427, 2007.

VISIOLI, L.J.; ENZWEILER, H.; KUHN, R.C.; SCHWAAB, M.; MAZUTTI, M.A. Technological prospection for biobutanol production. Recent Patents on Engineering, v. 7, p. 115-124, 2013.

YU, J.; ZHANG, T.; ZHONG, J.; ZHANG, X.; TAN, T. Biorefinery of sweet sorghum stem. Biotechnol Adv, v. 30, p. 811- 816, 2012. 